\title{
Significados y vivencias de los adultos responsables en el proceso de evaluación psicosociojudicial, en el Tribunal de Familia de Valparaíso
}

\author{
Alexis Bustos Villarroel*
}

\section{RESUMEN}

El presente trabajo es fruto de la investigación, enmarcada en el proceso de titulación del postítulo en Psicología, Familia y Derecho de la Universidad de Valparaíso. En ella se efectúa una investigación cualitativa exploratoria-descriptiva en torno a los significados y vivencias que se adquieren en el proceso de evaluación psicosociojudicial, en relación con adultos responsables ${ }^{1}$ que se encuentran ejerciendo los cuidados personales de $\mathrm{NNA}^{2}$, independiente de si éstos son hijos o no. La pregunta central de la investigación intenta indagar respecto de cuáles son los significados que otorgan los referentes parentales al proceso de evaluación psicosociojudicial. Los principales resultados, dicen relación con el acervo de conocimientos que posee la persona, los cuales se ven enriquecidos por los significados que se adquieren. De igual forma, la sensación de no ser oídos por la entidad judicial adquiere gran importancia.

Palabras Clave: Evaluación Psicosociojudicial - Significados - Vivencias Experiencia y Adultos Responsables

\section{Significados e vivências dos adultos responsáveis no processo de avaliação psicosociojudicial, no Tribunal de Família de Valparaíso}

\section{RESUMEM}

O presente trabalho é fruto da investigação, enquadrada no processo de titulação do pós-título em Psicologia, Família e Direito da Faculdade de Valparaíso. Nela se efetua uma investigação qualitativa exploratóriodescritiva em torno aos significados e vivências que se adquirem no

* Chileno. Asistente Social, Licenciado en Trabajo Social, Postítulo en Psicología Familia y Derecho, Postítulo en Psicología Jurídica al Servicio de la Investigación Forense y Criminal, Terapeuta Familiar Sistémico. Docente en AIEP en las carreras de Trabajo Social y Técnico en Trabajo Social. Adscrito a la Red comunal por la No Violencia Valparaíso. Actualmente Trabajador Social en Programa de Reparación de maltrato grave y abuso sexual, PRM CAVAS Regional de Valparaíso, convenio SENAME-PDI. Correo electrónico: bustosvillarroel@gmail.com

1 La categoría de adulto responsable, hace alusión a la madre, al padre, a ambos padres, terceros familiares o no familiares, incluyendo a los hogares residenciales proteccionales, quienes ejerzan los cuidados personales de los niños, niñas o adolescentes.

2 Niños, Niñas y Adolescentes. 
processo de avaliação psicosociojudicial, em relação com adultos responsáveis que se encontram exercendo os cuidados pessoais de NNA, independente si estes são filhos ou não. A pergunta central da investigação intenta indagar ao respeito de ¿quais são os significados que outorgam os referentes parentais ao processo de avaliação psicosociojudicial? Os principais resultados tem relação com o acervo de conhecimentos que possui a pessoa, os quais se vêm enriquecidos pelos significados que adquirem. Da mesma forma, a sensação de no serem ouvidos pela entidade judicial adquire uma grande importância.

Palavras Chave: Avaliação Psicosociojudicial - Significados - Vivências Experiência e Adultos Responsáveis

\section{Meanings and experiences of responsible adults in the psychological, social and judicial evaluation processes at the Valparaiso family court}

\section{ABSTRACT}

This work is part of the graduation process of students from the Postgraduate Diploma in Psychology, Family and Law at Universidad de Valparaíso. It consists of a qualitative, exploratory and descriptive research on meanings and experiences the psychological, social and judicial evaluation processes gain from adults in charge of the care of boys, girls and adolescents whether they are their own children or not. The study main results show that the knowledge heritage of the given person is enriched by the acquired meanings. In the same line, the feeling of not being heard by the judicial entity is of great importance.

Key words: Psychological, social and judicial evaluation - Meanings Experiences - Experiences and responsible adults

\section{Contexto}

El presente trabajo de investigación se ha efectuado en el contexto del área de protección de derechos de los NNA de SENAME ${ }^{3}$. Específicamente en la Diagnóstica, definida como una "Labor ambulatoria de asesoría técnica en el ámbito psicosocial y otros análogos a la autoridad judicial competente u otras instancias que lo soliciten" (DAM, SENAME, 2010). Esta Línea Diagnóstica Ambulatoria -en adelante DAM-, desarrolla un proceso de intervención efectuado con capacidad técnica especializada y contextualizado en el ámbito psicosociojurídico, evaluando vulneraciones de derechos vivenciadas por niños, niñas y adolescentes, lo cual se

3 Servicio Nacional de Menores, Chile. 
ve reflejado en la emisión de informes periciales proteccionales y/o penales, según corresponda. A este respecto, nos centramos en la labor concerniente a los Tribunales de Familia, centrando su accionar en determinar la presencia de maltrato grave en todas sus formas, delitos sexuales en todas sus formas, observación vicaria de violencia intrafamiliar, negligencia grave en el rol parental, ausencia de adulto responsable, abandono, descuido y cuidado personal.

Respecto de ello, la investigación se efectúa con padres, madres, familiares o cuidadores de NNA, que se encontraron vinculados al Tribunal de Familia de Valparaíso. Dicha vinculación obedece al establecimiento y/o resolución de alguna medida de protección vigente.

Debido a la experiencia adquirida en el desempeño de la labor pericial, surge la inquietud de responder a la pregunta general de investigación, la cual se relaciona con la experiencia y vivencia de los adultos responsables inmersos en el proceso. Esta intenta indagar sobre cuáles son los significados que otorgan los referentes parentales al proceso de evaluación psicosociojudicial. Para resolver esa interrogante, se plantearon los siguientes objetivos:

- Objetivo General de la Investigación: Indagar y describir cuáles son los significados que otorgan los referentes parentales al proceso de evaluación psicosociojudicial.

- Objetivos Específicos de la Investigación:

- Indagar y describir cuáles son los significados que otorgan los referentes parentales, respecto de la situación familiar en el proceso de evaluación psicosociojudicial.

- Indagar y describir los significados asociados a la evaluación psicosocial que efectúa el DAM, en relación a los adultos responsables de los NNA.

- Indagar y describir cuál es el significado que otorgan los referentes parentales al paso por el dispositivo jurídico.

\section{Fundamentos de la investigación}

Se tiene como premisa la importancia de "la noción de significado referido al acto significativo del individuo" (Salas, 2006), teniendo 
presente que cada vivencia y cada sentir ha de ser significado subjetivamente por el actor -miembro de la familia-, donde lo que se obtiene es la interpretación simbólica de la realidad. Al situar la conducta humana como una acción simbólica, la realidad social se complejiza y mediante el lenguaje los seres humanos construyen distintas realidades, diferentes explicaciones de los hechos, poniendo el acento desde "su" perspectiva (Bohannan, 1993:552-553).

Sobre lo mismo, tanto la vivencia de una vulneración de derechos, así como la participación dentro del proceso psicosociojudicial, estará inmersa en una serie de significados, los cuales son otorgados por cada actor social de la familia en cuestión. Así, es posible suponer que una experiencia en común (vulneración de derechos), será significada ya sea en forma particular como también en forma compartida por cada miembro de la familia.

\section{Sobre el proceso de evaluación psicosociojudicial}

Es necesario clarificar qué aspectos del proceso de evaluación psicosociojudicial han de ser considerados en el presente estudio, lo que se detalla a continuación:

1. Vulneración o sospecha de vulneración de derechos en NNA.

2. Ingreso de la demanda a tribunales de familia.

3. Audiencia preparatoria.

4. Ingreso a peritaje en el DAM.

a. Citación de las partes involucradas.

Considerados

b. Entrevistas psicológicas y sociales.

los puntos

$\mathrm{N}^{\circ} 2$ al $\mathrm{N}^{\circ} 5$

5. Audiencia de juicio en tribunal correspondiente.

6. Derivación del caso a los operativos sociales correspondientes.

\section{Sobre la metodología}

El proyecto de investigación se caracterizó por ser cualitativo y su nivel es exploratorio - descriptivo. De lo anteriormente expuesto, la investigación cualitativa se interesa por la vida de la gente, las experiencias vividas, los comportamientos, emociones y sentimientos (Strauss y Corbin, 2002:12). Como principal herramienta de recolección de información, se utilizó la entrevista semiestructurada, la cual "es más íntima, flexible y abierta" (Sampieri, 2006:597). 
Sobre la muestra, la presente investigación contempló la utilización de un Muestreo Teórico. Este se caracteriza por recurrir deliberadamente a personas o acontecimientos particulares, que maximicen la calidad de la información recopilada (Strauss y Corbin, 2002:219-220) "El muestreo teórico es importante cuando se exploran áreas nuevas o poco conocidas porque le permite al investigador escoger las perspectivas de muestreo que pueden producir el mayor rendimiento teórico". En el caso de la presente investigación, se solicitó la participación de adultos responsables de NNA que hayan estado vinculados al Tribunal de Familia de Valparaíso, debido a vulneraciones en los derechos fundamentales de los NNA a su cargo.

Finamente, el muestreo teórico tiene la característica de ser acumulativo. Cada acontecimiento vivenciado por los adultos participantes del estudio se suma al análisis final; se consideran las entrevistas de pre test y las entrevistas definitivas; ambas instancias en la investigación enriquecen las categorías que fueron formuladas posteriormente (Strauss y Corbin, 2002: 221).

Respecto del plan de análisis de los datos, está compuesto por el análisis de contenido (en adelante AC), el cual se refiere a cómo el texto funciona como un instrumento, el "contenido de un texto no es algo que estaría localizado dentro del texto en cuanto tal, sino fuera de él, en un plano distinto en relación con el cual ese texto define y revela su sentido" (Delgado y Gutiérrez, 1998:179). Así, el corazón del AC se caracteriza por denotar el contenido manifiesto como el contenido latente en los datos obtenidos a través de las entrevistas (Cáceres, 2003:57).

\section{Marco teórico: lo social y la vida cotidiana}

En las distintas realidades en las que participa el ser humano, las que se constituyen a través del lenguaje, insertas en relaciones cotidianas, es posible generar diversos consensos, los cuales conocemos como "realidad" y es, precisamente, en el espacio de lo cotidiano, en el cual se aprehende la realidad y se hace parte de la experiencia individual, la cual se estructura subjetiva y objetivamente a la vez (Berger y Luckmann, 1967:36 y ss).

Al respecto, Berger y Luckmann establecen que el mundo de la vida cotidiana, no sólo se da por establecido como realidad por los miembros de una sociedad, al compartir subjetivamente el significado de sus vidas, sino que "es un mundo que se origina en sus pensamientos y acciones, y que está sustentado como real por éstos" (Berger y Luckmann, 1967:36 y ss). 
Por otra parte, "entre las múltiples realidades existe una que se presenta como la realidad por excelencia. Es la realidad de la vida cotidiana. Aprehendo la realidad de la vida cotidiana como una realidad ordenada. La realidad de la vida cotidiana se presenta ya objetivada, o sea, constituida por un orden de objetos que han sido designados como objetos antes de que yo apareciese en escena" (Berger y Luckmann, 1967:37). Los autores plantean que es el lenguaje el que permite ordenar y dar sentido a la vida cotidiana; el lenguaje junto con el establecimiento de rutinas diarias, conforman y estructuran el sentido de realidad objetiva, realidad compacta y segmentada a la cual se tiene acceso desde el primer momento, puesto que ella existía con anterioridad a la persona misma.

A este respecto, Schütz (1993) utiliza el concepto de Mundo de la Vida para hacer alusión a la experiencia cotidiana del individuo, la cual se caracteriza por ser un mundo intersubjetivo, compartido, donde viven y conviven distintos sujetos, distintas subjetividades, las cuales van re-articulando las experiencias.

Estas experiencias y el nivel de conocimiento que de ellas emana, son de "sentido común, donde los elementos cognitivos que lo integran son parte de una matriz de conocimientos que llamamos experiencia del sentido común, y que está al alcance de todos los sujetos aunque diferenciada socialmente" (Salas, 2006:194).

Sobre el acápite anterior, es necesario tener presente las premisas básicas respecto del concepto de mundo de la vida y las experiencias que en él tienen ocurrencia:

- El Mundo de la Vida se refiere al mundo de la experiencia cotidiana y, por ende, cabe asumir que el sujeto es un actor que vivencia significativamente en este mundo.

- El sujeto está llamado a actuar y participar activamente en el mundo de la vida.

- El vivir, implicaría necesariamente actividad en el mundo.

En síntesis, el mundo de la vida, sería el entorno vital mismo desde el que se sitúa el sujeto humano, y cabe entender su relación con otros y con la naturaleza (Salas, 2006:174).

\section{Vivencias, experiencias y significados}

La característica del Mundo de la Vida, es que en ella se encuentra contenida la experiencia propia y la de otros individuos, y como consecuencia de ello, la presuposición de todos los procesos 
y fenómenos que ocurren en la vida cotidiana, es decir, los acontecimientos no se presentan de forma problemática o incognoscible; por el contrario, los elementos que conforman el mundo de la vida, su interacción y participación activa en él, son familiares y típicos a los individuos (Salas, 2006:194).

En este sentido, las personas disponen de un stock de elementos, de conocimientos. Este repertorio de posibilidades aprendidas mediante la experiencia va a posicionarse como el acervo de conocimientos o esquema de referencia, el cual es accesible para los sujetos que cohabitan en una sociedad o comunidad cualquiera.

El acervo de conocimientos es el recurso que posee el individuo y dispone en cualquier momento de él. Entonces, el acervo de conocimientos se expresaría como un esquema de referencia al que pertenece el "conocimiento de que el mundo en el que vivimos es un mundo más o menos bien determinado, con cualidades más o menos definidas, entre las cuales nos movemos, que se nos resisten y sobre las cuales podemos actuar" (Schütz, 1993:38).

El actuar en el mundo de la vida, cada paso en él y comprensión del mismo, en todo momento es un acervo de la experiencia previa, tanto las propias como las inmediatas (externas), experiencias que trasmiten otros individuos, los semejantes, sobre todo los padres, maestros, entre otros. Todo ese cúmulo experiencial está contenido en el acervo de conocimientos, el cual conforma el esquema de referencias que posibilita el actuar concreto en el mundo de la vida. Toda la experiencia y las significaciones de éstas, se relacionan indefectiblemente con ese esquema, de modo que los objetos y sucesos del mundo de la vida se presentan, desde el comienzo, en su carácter típico y cognoscible.

Los significados que se otorgan a una experiencia en particular, son de carácter subjetivo e intersubjetivo; así, cada experiencia vivenciada será significada por el actor particular, teniendo presente también la co-construcción por otros actores sociales. Independiente de ello, la experiencia tendrá un significado único, por lo tanto, una experiencia social o cultural nunca es, ni puede llegar a ser, lo mismo para dos personas (Bohannan, 1993:552553). Por cuanto el acervo de conocimientos de cada persona, le permite otorgar significaciones distintas a pesar de vivir una misma experiencia junto a otro. Ello es posible y mediado por su experiencia previa, como también por el esquema referencial 
que posea, que en definitiva, es la estructura significativa que le otorga a la vivencia.

Como epítome, el significado no deriva unidireccionalmente de los procesos cognitivos individuales, sino del proceso de interacción con otros sujetos en el mundo de la vida; de igual forma, según las significaciones presentes y pasadas, las cuales son compartidas por los miembros de una sociedad o comunidad en particular.

De esta manera, el significado que pueda adquirir la vivencia de enfrentar el proceso psicosociojudicial, va a variar en razón de cada familia e individuo que enfrente este proceso, puesto que la experiencia previa -o no- va a influir en cómo es significada esa vivencia.

\section{Resultados}

En relación al objetivo específico que dice relación con "Indagar y describir cuales son los significados que otorgan los referentes parentales, respecto de la situación familiar en el proceso de evaluación psicosociojudicial", los resultados obtenidos respecto de la aplicación del instrumento de recolección de datos permiten inferir que la situación familiar es significada por los referentes parentales respecto a la "responsabilidad en la crianza materna y paterna", es decir, referido a cuidados y protección inadecuados, así como el uso de medios correctivos abusivos por parte de los adultos responsables, vinculado específicamente a malos tratos hacia los NNA. Asimismo, los actores involucrados coinciden en que esta vivencia también es atribuida al consumo y abuso de drogas y alcohol por parte de los adultos responsables, señalándola como una "problemática individual" que afecta y produce descuido o trato negligente hacia los NNA. Los entrevistados señalan y comprenden la vulneración como "vivencias de abandono e indefensión" de los NNA, por parte de los referentes parentales, evidenciado principalmente en la institucionalización de los niños, niñas y adolescentes, como experiencia de daño y no de protección y cuidado que sustituyan la atención familiar.

Existe una significación compartida en asociar la vulneración en relación a "daño o vivencia traumática" y "transgresión de límites", caracterizado por situaciones de perjuicio, abuso físico o mental, descuido o trato negligente que, en situaciones descritas por ellos, es originada por familiares cercanos, por el o los adultos 
responsables. Cabe destacar que en los hallazgos obtenidos, los referentes parentales significan y resignifican la "importancia de escuchar a los niños" como un factor que disminuiría el riesgo de experimentar situaciones de vulneración hacia éstos, y que es destacado al momento de significar la vulneración como tal en comparación a tiempos anteriores. Junto a ello se señala además el "género" como factor no determinante al significar la vulneración en niños o niñas.

Surge como hallazgo individual y compartido en las y los sujetos evaluados, que el paso por el proceso de evaluación psicosociojudicial conlleva vivencias significativas a nivel familiar. Estas dicen relación con que se genera una reestructuración en la dinámica familiar, puesto que pasar por este proceso se posiciona como una crisis no normativa en el ciclo vital familiar, significándola como una experiencia traumática, generando por ejemplo un cuestionamiento en los roles materno y paterno respectivamente, sentimientos de culpa, lo que conlleva a una reflexión y posterior resignificación en torno al ejercicio del rol. Finalmente, los entrevistados y entrevistadas le otorgan utilidad al proceso psicosociojudicial, generando aprendizajes familiares significativos, los que tienen impacto directo en la comunicación familiar.

Sobre el objetivo específico de la presente investigación, que dice relación con "Indagar y describir los significados asociados a la evaluación psicosocial que efectúa el DAM, en relación a los adultos responsables de los NNA", los resultados obtenidos se categorizaron en dos apartados, uno de los cuales dice relación con las sensaciones experimentadas por los adultos responsables antes, durante y después de la evaluación en el DAM. Por otra parte, se obtuvo información relacionada con el "Ser Oído", como contraposición a lo que ocurre en el contexto del Tribunal de Familia.

En lo que concierne a la categoría de las "Sensaciones", se pudo constatar que la primera de ellas se relaciona con el sentimiento de angustia y tensión inicial; ello se relaciona con el paso anterior -en muchos de los casos-, por el contexto del Tribunal, el cual es significado como un ambiente hostil e intimidante. Con esa experiencia, las personas que pasan por la evaluación del DAM presuponen que el ambiente será el mismo experimentado en el Tribunal, es por ello que la etapa previa a la evaluación es significada como tensionante en sí misma, sumado a ello, la 
exigencia de tener que ser "aprobado" por un organismo externo, contribuye a la maximización negativa de la experiencia. En la mayoría de los casos entrevistados, la aprobación externa es un tópico que se reitera, dado que de esta aprobación depende, en muchos casos, la resolución final esgrimida por el Juez de Familia.

Si bien, la sensación inicial es de tensión y angustia, ésta cambia al momento de ser recibidos en el espacio DAM, considerando la distribución espacial de la oficina, como también el trato de los profesionales hacia los adultos próximos a entrevistar. En ello, los entrevistados identifican claramente una diferencia entre los ambientes y el trato del Tribunal respecto del trato en el DAM. Por una parte, la experiencia vivida en el Tribunal es significada con calificativos negativos e incluso peyorativos, por su parte, los entrevistados informan que la tensión y angustia inicial se diluye al momento de comenzar la entrevista psicosocial. Ello, porque se sienten escuchados y la instancia les sirve de espacio de desahogo, a pesar de tener claridad que el espacio de evaluación psicosociojudicial se relaciona directamente con el Tribunal de Familia y con la futura resolución judicial.

Esta sensación de desahogo se encuentra acompañada con la vivencia de abrirse a extraños, los cuales van a tomar determinaciones sobre la vida futura de los NNA involucrados en el proceso. Conjuntamente con ello, la entrevista misma produce sensaciones adversas, ya que por una parte, la experiencia se significa como ser escuchado y ser tratado de forma distinta que en el Tribunal, aunque de igual forma, se tomarán decisiones importantes y vitales para la familia y los NNA a su cargo. Si bien, en la mayoría de los entrevistados, la resolución judicial fue positiva, presentan temor al espacio de evaluación, ya que ignoran qué es lo que se está evaluando. Esta situación se enmarca en otra sensación adversa, ya que la entrevista efectuada en el DAM es significada como un espacio de aprendizaje y un proceso reflexivo para revisar sus propias historias vitales y cómo ello podría haber afectado el normal funcionamiento de la familia.

En lo que respecta a la categoría "Ser Oído", los participantes de la presente investigación rescatan y significan la experiencia de la entrevista psicosocial efectuada en el DAM, como un espacio donde son escuchados y se les permite desahogarse. Este desahogo dice relación con la imposibilidad de explicar su vivencia en el espacio de audiencia preparatoria en el Tribunal. Como contraposición a ello, el espacio de entrevista DAM, facilita 
la expresión individual respecto de la vulneración de derechos, la historia individual y familiar.

Nuevamente, la comparación entre los ambientes Tribunal DAM cobra relevancia, esta vez no en las sensaciones vivenciadas en ambos espacios, sino en la capacidad de los profesionales de escuchar, o posibilitar el diálogo -Ser Oído-, frente a la incapacidad de comunicar lo que se quiere decir -(No) Ser Oído-. Esta dualidad en la capacidad de escucha, pone en evidencia la diferencia percibida en los ambientes, si bien, la mayoría de los entrevistados puede comprender que el espacio de Tribunal es una instancia seria y burocrática, no logran comprender por qué no se les escucha en dicha instancia, significando esa vivencia como adversa, cortante, culpabilizante, donde "no se tiene derecho ni a voz ni a voto".

Respecto al objetivo específico de la presente investigación, que dice relación con "Indagar y describir cuál es el significado que otorgan los referentes parentales al paso por el dispositivo jurídico", los resultados obtenidos frente a la aplicación del instrumento de recolección de datos, arrojaron que al abordar la temática "Tribunal", los sujetos presentan una vivencia compartida o intersubjetiva ante el paso por dicha instancia.

Los resultados, en general, permiten inferir que el paso por esta instancia es significada por los referentes parentales como un momento "traumático y frustrante", el cual se puede clasificar en distintas esferas.

En primera instancia, que dice relación al "Proceso", los actores coinciden en manifestar que el proceso judicial en sí se torna engorroso, con poca claridad del procedimiento, con existencia de una rigidez del sistema y escasa empatía por parte de los funcionarios de dicha entidad, lo cual vivencian en la interacción con los distintos funcionarios, desde el Juez o Magistrado, pasando por los abogados, Consejeros Técnicos y administrativos.

En segunda instancia, la que dice relación con las "Sensaciones", los actores coinciden en manifestar a través de su discurso diferentes sensaciones, aludiendo en términos generales a una experiencia de intimidación y prejuicio, donde principalmente el cuestionamiento y/o enjuiciamiento por parte del sistema, apunta a su ser individual, a su rol paterno o materno y a su capacidad como referente parental responsable y capaz. Es relevante destacar que, si bien los sujetos entrevistados no 
participan de un proceso penal, su experiencia compartida es de sentirse tratados como delincuentes y culpables de "algo".

En tercera instancia, relacionada con la subcategoría de "No Ser Oído", los actores coinciden en manifestar la falta de escucha por parte del operador judicial, significándola como una experiencia de "falta de tiempo y un ambiente cortante"; en suma, su vivencia es haber sido invisibilizados como participantes del proceso judicial, sin ser tomados en cuenta frente a las resoluciones finales.

En última instancia, la cual dice relación con el "Maltrato Institucional", los actores coinciden en describir el espacio judicial como "un contexto vulnerador y un ambiente hostil”, atribuyéndole además un carácter maltratador a dicha experiencia, la cual principalmente está caracterizada por la insensibilidad que perciben de parte de los funcionarios, donde su mayor vivencia es sentirse ajenos al sistema dado el constante encasillamiento negativo por parte de los operadores judiciales, como por ejemplo, "sentirse todos dentro del mismo saco".

Es posible extrapolar entonces, ante los hallazgos extraídos de esta investigación, los siguientes cuestionamientos: ¿Qué sucede con el principio de desformalización del proceso? ¿Qué sucede con el procedimiento oral por oposición a lo escrito? ¿Qué sucede con el principio de inmediación de las causas? ¿Qué pasa con el principio de colaboración entre las partes y/o actores del proceso? En suma, ¿qué sucede con los principios rectores de los Tribunales de Familia?

Si bien existen significados relacionados con la categoría Tribunal, que tienen que ver con el funcionamiento interno de dicha entidad, surge una clara diferencia entre el trato que ejerce un magistrado masculino, al trato que ejerce un magistrado femenino, lo cual es significado por la vivencia particular y compartida por las entrevistadas, quienes sentían cuestionado su rol materno y protector por parte de las magistradas. Por otro lado, significan la vivencia del ejercicio del magistrado con una mayor empatía hacia su situación particular. Por último, existe de parte de las entrevistadas un "prejuicio" hacia las magistradas, el cual se relaciona con el trato que esperan recibir por el hecho de compartir género (buen trato). 


\section{Conclusiones}

Al finalizar el presente proceso de investigación social, ha quedado de manifiesto la doble falencia que presenta el operar del Tribunal de Familia; por una parte, el maltrato institucional que se experimenta al asistir a dicha instancia, se posiciona como una experiencia significada negativamente, inmersa en una serie de sensaciones desagradables para los usuarios del sistema judicial. Esta situación pone en alerta el trato recibido por profesionales y funcionarios de la entidad pública, que a todas luces, trasgreden la integridad de quienes han llegado a la instancia judicial.

La evidencia recopilada aquí, sugiere explícitamente que se revisen las prácticas profesionales y no profesionales de quienes ofician en el poder judicial, puesto que no solo trasgreden a los adultos responsables de NNA, sino que vulneran nuevamente la integridad y los derechos de los niños, existiendo así un funcionamiento inadecuado. Ya que, no es posible que la instancia judicial que está llamada a proteger y promocionar los Derechos de los Niños, Niñas y Adolescentes -consagrados en la CIDN-, trasgreda los mismos derechos que dice proteger. Esta contradicción vital se visualiza en la sobreexposición a contextos judiciales o las dobles y triples evaluaciones que se efectúan, victimizando por segunda o tercera vez a los NNA que ya han sido vulnerados en primera instancia.

La segunda falencia detectada tiene directa relación con el funcionamiento propio del procedimiento judicial en Familia, el cual debiera ceñirse estrictamente a lo expuesto en la ley $\mathrm{N}^{\circ} 19.968$-que crea los tribunales de Familia y especifica su funcionamiento-, dicha ley tiene por objeto, entre otras cosas, simplificar y hacer accesible el procedimiento a los usuarios. Pero contrariamente a ello, la experiencia vivida en el Tribunal, es significada como engorrosa y burocrática en lo extremo, principios que se pretendían abolir con la entrada en vigencia de la ley antes mencionada.

Esta incoherencia detectada entre lo que plantea la Ley y lo que ocurre en el Tribunal puede tener su génesis en los funcionarios mismos, puesto que se ha intentado mejorar y agilizar el procedimiento en Familia, re-organizando el funcionamiento y las materias que está llamado a conocer. 
Los funcionarios, en gran medida, prosiguieron del sistema anterior -del sistema de tribunal de "menores"-, por lo cual y por diversos motivos puede que no hayan actualizado los conocimientos para actuar y proceder bajo el nuevo escenario. Independiente de las capacitaciones que efectúa el poder judicial, con el objeto de profesionalizar y actualizar los conocimientos en sus funcionarios, hay que tener presente que un número importante de funcionarios prosiguió del sistema antiguo al nuevo y el "habitus" adquirido bajo el viejo paradigma puede ser imperturbable ante las nuevas exigencias.

De igual forma, se hace necesario obtener información sistemática, de primera fuente, desde los actores del operativo judicial. Esto es, desde el Tribunal de Familia, puesto que la información recopilada aquí pertenece sólo a una parte involucrada en el proceso de evaluación psicosociojudicial.

Es por ello que es de suma importancia recoger la apreciación de los Magistrados, Consejeros Técnicos, Encargados de Sala, Encargados de Audio y en general de todos los funcionarios que se desempeñan en el Tribunal de Familia. Ello dará una visión in situ de los fenómenos aquí expuestos y, muy probablemente, surjan nuevos hallazgos y nuevas líneas de investigación.

\section{Bibliografía}

Berger, T.; Luckmann, P. (1968). La Construcción Social de la Realidad. Buenos Aires: Amorrortu Editores.

Bohannan, P. (1993). Antropología, lecturas.(2a ed.). España: Editorial McGraw-Hill.

Cáceres, P. (2003). Análisis Cualitativo de Contenido: una alternativa metodológica alcanzable, Psicoperspectivas individuo y sociedad, 2(1), 53-82.

Delgado, J.; Gutiérrez, J. (1998). Métodos y técnicas cualitativas de investigación en ciencias sociales. Editorial Síntesis.

Ministerio de Justicia, Servicio Nacional de Menores (Minuta Técnica Sobre Línea Diagnóstico, Proyectos De Diagnóstico Ambulatorio DAM)

Ministerio de Justicia, Servicio Nacional de Menores. Formulario de Presentación de proyectos, línea diagnóstico, proyectos de Diagnóstico ambulatorio DAM PILLELTU Valparaíso. 
Sampieri, R.; Fernández, C. y Baptista, P. (2006). Metodología de la Investigación (4a ed.). México D.F: McGraw-Hill/ Interamericana editores, S.A.

Schütz, A. (1993). La Construcción significativa del mundo social. Barcelona: ediciones Paidós.

Strauss, A.; Corbin, J. (2002). Bases de la Investigación Cualitativa, técnicas y procedimientos para desarrollar la teoría fundamentada ( $1^{\text {a }}$ ed. en español). España: editorial Universidad de Antioquia.

Salas, R. (2006). El mundo de la vida y la fenomenología sociológica de Schütz. Apuntes para una filosofía de la experiencia. Revista de filosofía, 15, 167-199. Disponible en http://dialnet.unirioja.es/servlet/ articulo?codigo $=2293997$ 